\title{
Modified release prednisone in patients with rheumatoid arthritis
}

\author{
J W G Jacobs, J W J Bij|sma
}

Since the demonstration of the potent anti-inflammatory and immunosuppressive effects of glucocorticoids (GCs) halfway through the previous century, GCs have been the most frequently and generally used anti-inflammatory and immunosuppressive class of drugs in a wide spectrum of immune-mediated diseases such as systemic autoimmune diseases, including the arthritides like rheumatoid arthritis (RA), vasculitides and allergic conditions. Their low cost has enabled their application worldwide. The rationale for their use in active RA is the fast symptomatic relief through inhibition of the inflammatory process. ${ }^{1}$ In addition, in the last decades GCs have also been shown to inhibit radiographic joint damage in early RA, which led to the paradigm shift that GCs are disease-modifying antirheumatic drugs (DMARDs). ${ }^{2} 3$ In modern treatment strategies which aim for fast remission in patients with early RA (tight control strategies), GCs are often used in combination with other DMARDs. ${ }^{4} 5$ Although the adverse effect (AE) spectrum of low- to medium-dose GCs seems to be modest, ${ }^{6}$ AEs of GCs have been an issue for many years. Research has been aimed at decreasing the risk of AEs of GCs and at improving the therapeutic ratio of this class of drugs. In this paper, after a short description of developments to improve the therapeutic ratio of GCs, we will discuss modified-release prednisone (MR prednisone) and the study published in this issue on this new drug formulation.?

\section{DEVELOPMENTS TO IMPROVE THE THERAPEUTIC RATIO OF GCS}

In addition to guidelines to improve the clinical use of existing $\mathrm{GCs},{ }^{8}$ new formulations have been and are being developed to improve the therapeutic ratio of GCs.

University Medical Center Utrecht, Utrecht, The Netherlands

Correspondence to J W G Jacobs, Department of Rheumatology and Clinical Immunology, F02.127, University Medical Center Utrecht, PO Box 85500 , 3508 GA Utrecht, The Netherlands; j.w.g.jacobs@ umcutrecht.nl
Deflazacort, ${ }^{9}$ an oxazoline derivative of prednisolone, was initially thought to be as effective as prednisone while inducing fewer AEs, but there was a problem with the real equivalence ratio compared with prednisone $e^{10}$ and this drug has not become a major breakthrough. Knowledge about the mechanisms of GCs leading to beneficial effects (predominantly by the genomic effects of transrepression) and to AEs (predominantly by the genomic effects of transactivation) led to the development of selective GC-receptor agonists (SEGRAs) or dissociating GCs. ${ }^{11}$ However, a major limiting factor in comparing AEs of SEGRAs with those of conventional GCs is that precise data on the frequency and severity of AEs of GCs and methods on how to assess them are, to a large extent, lacking. ${ }^{12}$ Initiatives to improve monitoring and documentation of AEs of GCs have recently been developed. ${ }^{8}$ GC preparations releasing nitric oxide, the so-called nitrosteroids, could induce stronger anti-inflammatory effects because nitric oxide also has antiinflammatory effects. ${ }^{13}$ These drugs could have an improved therapeutic ratio but have yet to be tested in patients. The combination of prednisolone and dipyridamole has been reported to boost and extend the net GC effect in laboratory models. ${ }^{14}$ The next step is to demonstrate an improved therapeutic ratio in patients in adequate comparative clinical trials, assessing predefined beneficial effects and AEs in a standardised manner. ${ }^{15}$ Liposomes containing GCs and targeted to integrins expressed on endothelial cells at sites of inflammation, which deliver their GC specifically to these sites, have been studied. ${ }^{16}$ Their selective biodistribution might enable less frequent and lower dosing which could result in an improved therapeutic ratio. The safety of liposomal prednisolone has been evaluated in a small group of patients with RA and the results (published as an abstract) seem promising. ${ }^{17}$

Based on the cyclical variability of biological processes (chronobiology), an MR prednisone tablet has recently been developed to increase the therapeutic ratio of prednisone in $\mathrm{RA}$. In this issue of the journal, Buttgereit et al describe the results of a 9-month open-label extension of a 3-month double-blind trial published earlier. ${ }^{7}$

\section{CHRONOBIOLOGY IN RA}

In healthy controls, plasma cortisol levels exhibit a circadian (approximately $24 \mathrm{~h}$ ) rhythm: they start to rise in the early hours of the morning, peak around 06:00-08:00 $\mathrm{h}$ and have a nadir around 22:00-02:00 h, after which they start to rise again. Patients with active RA have an earlier rise, starting at 23:0002:00 h, ${ }^{18} 19$ and a higher peak (figure 1). Nevertheless, it is suggested that this increased secretion of cortisol in RA is insufficient in view of the arthritis activity. ${ }^{18}$ This earlier rise is preceded and possibly caused by a rise in the proinflammatory cytokine interleukin 6 (IL-6), ${ }^{19}$ which plays a pleiotropic role in the pathogenesis of RA including the typical pattern of joint pain, swelling and stiffness which are most severe on waking. IL- 6 also stimulates the hypothalamicpituitary-adrenal (HPA) axis which leads to increased levels of cortisol and suppression of arthritis (see figure 1). Early morning stiffness is characteristic of inflammation in RA, and its duration and severity are measures of disease activity. Both IL-6-targeting therapy, ${ }^{20}$ which has been shown to suppress disease activity and prevent joint destruction in RA, and the present study suggest that this pathophysiological model applies. ${ }^{21}$

To try to turn this biorhythm to advantage, an earlier study showed that low doses of prednisolone taken at 02:00 h had more effect on severe morning symptoms of RA than the same dose at 07:30 h. ${ }^{22}$ However, patients had to be woken up for their medication at 02:00 h which itself will influence the biorhythm and HPA axis.

\section{MR PREDNISONE}

The newly-developed MR prednisone releases prednisone about $4 \mathrm{~h}$ after ingestion. By taking it in the evening and thus adapting its release to the circadian increases in proinflammatory cytokine concentrations, the symptoms of RA early in the morning could be less than when the same dose of prednisone is taken early in the morning. In a 3-month double-blind trial, patients with a duration of morning stiffness $\geq 45 \mathrm{~min}$, a pain score of $\geq 30 \mathrm{~mm}$ on a $100 \mathrm{~mm}$ visual analogue scale, $\geq 3$ painful joints, $\geq 1$ swollen joint(s) and an erythrocyte sedimentation 


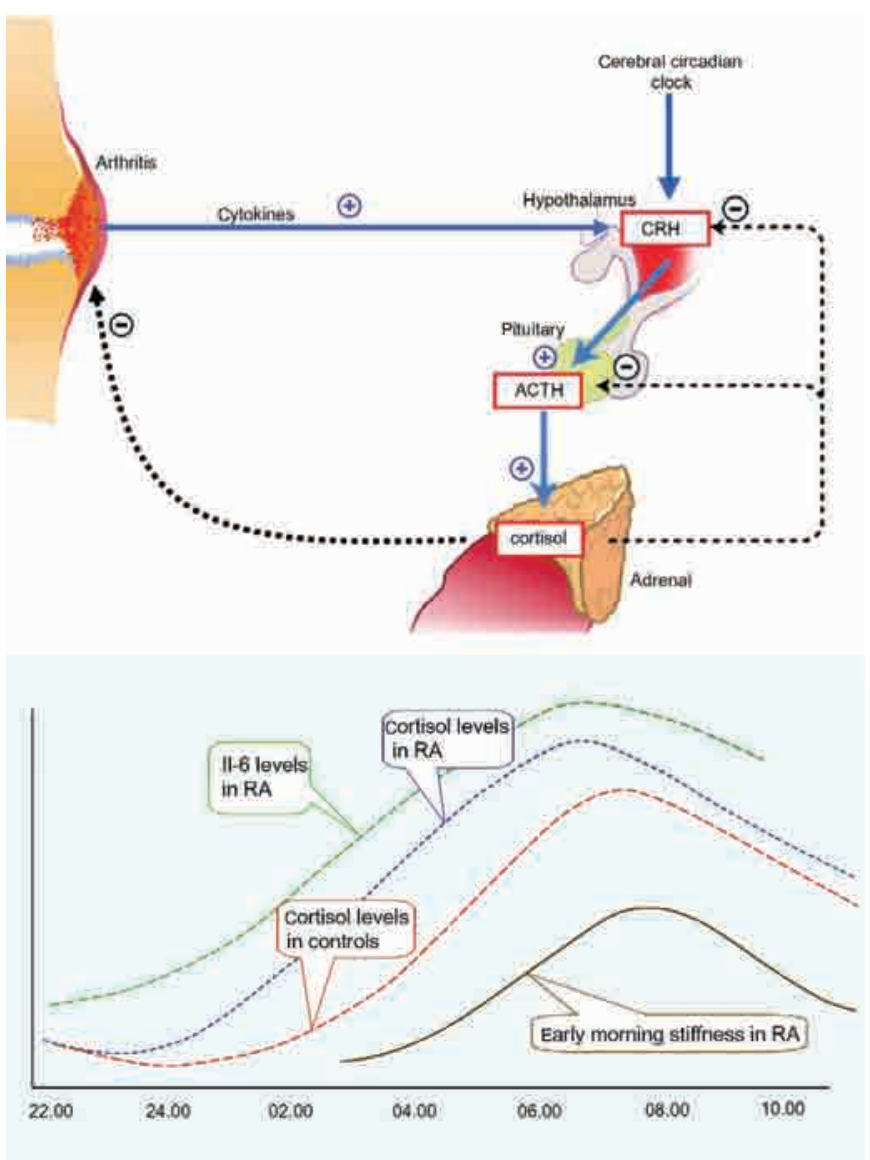

Figure 1 Diagram of chronological biological processes in rheumatoid arthritis (RA) compared with those in healthy controls. ACTH, adrenocorticotrophic hormone; $\mathrm{CRH}$, corticotrophinreleasing hormone.

rate $\geq 28 \mathrm{~mm}$ or $\mathrm{C}$ reactive protein concentration $\geq 1.5$ times the upper limit of normal who were on $\mathrm{GC} \geq 3$ months with a stable daily dose of $2-10 \mathrm{mg}$ prednisone equivalent for $\geq 1$ month were included. In a double-dummy manner, patients were randomised either to continue their prednisone or to switch to MR prednisone. At the end of the trial the difference in duration of morning stiffness between the two groups was about $30 \mathrm{~min}$ in favour of MR prednisone. IL-6 levels decreased significantly in the group using MR prednisone compared with the levels in the prednisone group. Remarkably, there were no differences in the other assessed variables of disease activity between the two groups. The safety profile did not differ between treatments, ${ }^{23} 24$ but no checklists with predefined AEs were used for scoring them.

In the open extension which added 9 months to the 3 -month trial, the patients in the group allocated to continue the prednisone were also switched to MR prednisone. The duration of morning stiffness in these patients also improved. At 12 months, both former groups showed a decrease in IL-6 level to 50\%, a decrease in VAS pain of about $10 \mathrm{~mm}$ and a decrease in DAS28 of 1 unit. About one-third of patients achieved an ACR20 response. AEs were comparable to those of historical controls.

\section{WHAT DOES THIS 9-MONTH OPEN-LABEL EXTENSION SHOW?}

First, the effect of MR prednisone on morning stiffness and IL- 6 levels in patients with RA already on GC is corroborated as these effects were also seen in the former prednisone group after switching to MR prednisone. Second, it shows that the beneficial effect on morning stiffness and the reduction in IL- 6 levels are sustained up to 12 months. Third, it demonstrates the difficulty in interpreting clinical results of trials or extensions that are not double-blind. We will discuss this, looking in detail at figure 2 in the paper by Buttgereit et al. ${ }^{7}$ In the group originally randomised to MR prednisone, the effect on morning stiffness seemed to stabilise after 2 months at a decrease of about $35 \%$ but, after the switch at 3 months to the open-label study, a further decrease of about $20 \%$ was seen at month 6 . The total decrease equals the fall in duration of morning stiffness in the group switching to the open-label study at 3 months from prednisone to MR prednisone (about 55\%). In this latter group, however, this decrease is already seen after 3 months and, in the former group, only after 6 months, of which 3 months is open label. Stabilisation of the effect in the original MR prednisone group at 2 months and the 3 -month difference between the groups in the period to achieve the maximal reduction in morning stiffness are compatible with a placebo effect during the open-label phase of about $20 \%$ further improvement in morning stiffness. An alternative explanation could be changes in the medication as, in the open-label extension, medication was free. Although the authors report that no important changes in medication were recorded, concomitant non-steroidal anti-inflammatory drug use and even non-pharmacological therapies may influence the clinical effect of GCs. ${ }^{25}$ Either way, it is difficult to attribute the full effect on morning stiffness at 6 months to MR prednisone alone (a smaller part of the effect might be attributed to a placebo effect) and to appreciate the other modest beneficial clinical effects during the open-label phase, especially because these effects were not present in the double-blind phase of the study. The sustained reduction in IL- 6 levels up to 12 months was, of course, not subject to a placebo effect.

\section{WHAT DO WE KNOW IN ADDITION?}

MR prednisone decreased levels of IL- 6 in comparison with the continued use of prednisone. IL-6, next to IL-1 and tumour necrosis factor $\alpha$, stimulates the adrenal glands to secrete cortisol via the HPA axis (see figure 1).$^{18}$ Theoretically, this further decrease in IL-6 levels could result in a greater risk of $M R$ prednisone inducing HPA axis suppression than prednisone. The authors state that there were no signs or symptoms to indicate any aggravation of HPA axis suppression in their study, but this is clinically difficult to detect during ongoing GC therapy. However, an analysis of corticotrophin-releasing hormone tests in a subgroup of 28 patients at three time points during the total 12 -month study period (3 months double-blind and 9 months open-label) has been reported, and no sign of increased adrenal impairment on treatment with MR prednisone was observed. ${ }^{26}$

\section{WHAT DO WE NOT YET KNOW?}

Although there seems no clear reason to assume MR prednisone would be very 
different from prednisone in inhibiting the development of radiological joint damage, the DMARD properties of MR prednisone in RA should be investigated. The same applies to other disease and outcome variables in RA. At the EULAR 2010 Congress, a study was reported in patients with active RA not receiving GC therapy and with an inadequate response to DMARDs starting concomitant $5 \mathrm{mg}$ $\mathrm{MR}$ prednisone daily or placebo. After 12 weeks a statistically significantly and clinically relevantly higher response rate (ACR20 and ACR50 criteria) was found in the MR prednisone group compared with the placebo group (49\% vs $29 \%$ and $23 \%$ vs $9 \%$, respectively). However, we do not know whether the effect of prednisone in this situation would have been less. It would be interesting to assess the effects of MR prednisone versus those of prednisone in other rheumatological and non-rheumatological diseases such as polymyalgia rheumatica and Crohn's disease. Furthermore, the spectrum of long-term AEs is important. To discriminate between the AEs of MR prednisone and those of prednisone, standardised scoring in large groups of patients using a predefined AE list is necessary. ${ }^{8}$

\section{CONCLUSION}

Although in our view the superior beneficial effects of MR prednisone compared with those of prednisone on clinical disease and outcome variables other than morning stiffness have not yet been firmly established, for patients with RA on low to medium doses of prednisone who still experience a long duration of morning stiffness, MR prednisone seems to be a valuable new asset which reduces the duration of stiffness to a clinically relevant extent. The new drug is clearly superior to prednisone in this respect.

Competing interests JWJB received consultancy fees from Nitec.

Provenance and peer review Commissioned; externally peer reviewed.

Accepted 12 May 2010
Arch Dis Child 2010;69:1257-1259.

doi:10.1136/ard.2010.132738

\section{REFERENCES}

1. Saag KG, Criswell LA, Sems KM, et al. Low-dose corticosteroids in rheumatoid arthritis. A metaanalysis of their moderate-term effectiveness. Arthritis Rheum 1996;39:1818-25.

2. Bijlsma JW, Hoes JN, Van Everdingen AA, et al. Are glucocorticoids DMARDs? Ann N Y Acad Sci 2006;1069:268-74.

3. Kirwan JR, Bijlsma JW, Boers M, et al. Effects of glucocorticoids on radiological progression in rheumatoid arthritis. Cochrane Database Syst Rev 2007;1:CD006356.

4. Bakker MF, Jacobs JW, Verstappen SM, et al. Tight control in the treatment of rheumatoid arthritis: efficacy and feasibility. Ann Rheum Dis 2007;66(Suppl 3):iii56-60.

5. Hoes JN, Jacobs JWG, Buttgereit F, et al. Current view of glucocorticoid co-therapy in rheumatoid arthritis. Nat Rev Rheumatol 2010;(In Press).

6. Da Silva JA, Jacobs JW, Kirwan JR, et al. Safety of low dose glucocorticoid treatment in rheumatoid arthritis: published evidence and prospective trial data. Ann Rheum Dis 2006;65:285-93.

7. Buttgereit F, Doering G, Schaeffler A, et al. Targeting pathophysiological rhythms: prednisone chronotherapy shows sustained efficacy in rheumatoid arthritis. Ann Rheum Dis 2010;69:1275-80.

8. Goes van der MC, Jacobs JWG, Boers M, et al. Monitoring adverse events of low-dose glucocorticoids therapy: EULAR recommendations for clinical trials and daily practice. Ann Rheum Dis 2010; (In Press).

9. Eberhardt R, Krüger K, Reiter W, et al. Longterm therapy with the new glucocorticosteroid deflazacort in rheumatoid arthritis. Double-blind controlled randomized 12-months study against prednisone. Arzneimittelforschung 1994;44:642-7.

10. Saviola G, Abdi Ali L, Shams Eddin S, et al. Compared clinical efficacy and bone metabolic effects of low-dose deflazacort and methyl prednisolone in male inflammatory arthropathies: a 12-month open randomized pilot study. Rheumatology (Oxford) 2007:46:994-8.

11. Buttgereit F, Burmester GR, Lipworth BJ. Optimised glucocorticoid therapy: the sharpening of an old spear. Lancet 2005;365:801-3.

12. Hoes JN, Jacobs JW, Verstappen SM, et al. Adverse events of low- to medium-dose oral glucocorticoids in inflammatory diseases: a metaanalysis. Ann Rheum Dis 2009;68:1833-8.

13. Paul-Clark MJ, Mancini L, Del Soldato P, et al. Potent antiarthritic properties of a glucocorticoid derivative, NCX-1015, in an experimental model of arthritis. Proc Natl Acad Sci USA 2002;99:1677-82.

14. Zimmermann GR, Avery W, Finelli AL, et al. Selective amplification of glucocorticoid anti- inflammatory activity through synergistic multitarget action of a combination drug. Arthritis Res Ther 2009;11:R12.

15. Jacobs JW, Bijlsma JW. Innovative combination strategy to enhance effect and diminish adverse effects of glucocorticoids: another promise? Arthritis Res Ther 2009;11:105.

16. Koning GA, Schiffelers RM, Wauben MH, et al. Targeting of angiogenic endothelial cells at sites of inflammation by dexamethasone phosphate-containing RGD peptide liposomes inhibits experimental arthritis. Arthritis Rheum 2006;54:1198-208.

17. Barrera P. Long-circulating liposomal prednisolone versus pulse intramuscular methylprednisolone in patients with active rheumatoid arthritis. Arthritis Rheum 2008;58(Suppl):S453 (abstract).

18. Crofford LJ, Kalogeras KT, Mastorakos G, et al. Circadian relationships between interleukin (IL)-6 and hypothalamic-pituitary-adrenal axis hormones: failure of IL-6 to cause sustained hypercortisolism in patients with early untreated rheumatoid arthritis. $J$ Clin Endocrinol Metab 1997;82:1279-83.

19. Straub RH, Cutolo M. Circadian rhythms in rheumatoid arthritis: implications for pathophysiology and therapeutic management. Arthritis Rheum 2007:56:399-408.

20. Maini RN, Taylor PC, Szechinski J, et al.; CHARISMA Study Group. Double-blind randomized controlled clinical trial of the interleukin- 6 receptor antagonist, tocilizumab, in European patients with rheumatoid arthritis who had an incomplete response to methotrexate. Arthritis Rheum 2006:54:2817-29.

21. Nishimoto N, Hashimoto J, Miyasaka N, et al. Study of active controlled monotherapy used for rheumatoid arthritis, an IL-6 inhibitor (SAMURAI): evidence of clinical and radiographic benefit from an $\mathrm{x}$ ray reader-blinded randomised controlled trial of tocilizumab. Ann Rheum Dis 2007;66:1162-7.

22. Arvidson NG, Gudbjörnsson B, Larsson A, et al. The timing of glucocorticoid administration in rheumatoid arthritis. Ann Rheum Dis 1997; 56:27-31.

23. Buttgereit F, Doering G, Schaeffler A, et al. Efficacy of modified-release versus standard prednisone to reduce duration of morning stiffness of the joints in rheumatoid arthritis (CAPRA-1): a double-blind, randomised controlled trial. Lancet 2008;371:205-14.

24. Bijlsma JW, Jacobs JW. Glucocorticoid chronotherapy in rheumatoid arthritis. Lancet 2008;371:183-4.

25. van Everdingen AA, Siewertsz van Reesema DR, Jacobs JW, et al. The clinical effect of glucocorticoids in patients with rheumatoid arthritis may be masked by decreased use of additional therapies. Arthritis Rheum 2004;51:233-8.

26. Alten R, Doering G, Witte $S$, et al. Hypothalamuspituitary-adrenal (HPA)-axis function in rheumatoid arthritis patients treated with either standard or modified-release prednisone. Arthritis Rheum 2009;60(Suppl):S148 (abstract). 
J W G Jacobs and J W J Bijlsma. Modified release prednisone in patients with rheumatoid arthritis. Ann Rheum Dis 2010;69:1257-1259. The incorrect journal abbreviation has been published: Arch Dis Child 2010;69:1257-1259.doi:10.1136/ard.2010.132738. The correct journal abbreviation should be Ann Rheum Dis 2010;69:1257-1259. doi:10.1136/ard.2010.132738.

doi:10.1136/ard.2010.132738corr1 